\title{
Development of visible and NIR imaging equipment for small animals with smart pad
}

\author{
Nyeon Sik Eum ${ }^{\mathrm{a}}$, Jung Hyun Han ${ }^{\mathrm{a}}$, Ki Woong Seong ${ }^{\mathrm{b}}$, Jong Ha Lee ${ }^{\mathrm{c}}$ and Hee Joon Park ${ }^{\mathrm{c}, *}$ \\ ${ }^{a}$ U-BioMed Inc., B. I. Center, Kyungpook National University, 80 Daehak-ro, Buk-Gu, Daegu, 704- \\ 701, Korea \\ ${ }^{b}$ Department of Biomedical Engineering, Kyungpook National University Hospital, 130 Dongdeok-ro, \\ Jung-Gu, Daegu, 704-712, Korea \\ ${ }^{c}$ Department of Biomedical Engineering, Keimyung University, 1095 Dalgubeoldae-ro, Dalseo-Gu, \\ Daegu, 702-701, Korea
}

\begin{abstract}
The portable visible and near-infrared (NIR) imaging equipment for a pre-clinical test with small animals was designed and developed in this paper. The developed equipment is composed of a CCD camera, a focusing lens, an objective lens, a NIR band pass filter and a NIR filter driving motor. An NIR ray is mainly used for imaging equipment because it has high light penetration depth in biological tissue. Therefore, NIR fluorescent agents are available for chemical conjugation to targeting molecules in vivo. This equipment can provide a visible image, NIR image and merged image simultaneously. A communication system was specifically established to check obtained images through a smart pad in real time. It is less dependent on space and time than the conventional system.
\end{abstract}

Keywords: Visible/NIR image, animal imaging, fluorescence, mobile-based, merged image

\section{Introduction}

Development processes (new drug, biomarker, etc.) need too much money and a long time. Preclinical tests are required to verify the effectiveness of new drugs or bio-markers [1,2]. In the process of a preclinical test, researchers normally use imaging equipment with small animals. This equipment provides biological imaging of biotransformation to researchers.

There are many types of imaging equipment such as optical imaging, nuclear imaging, magnetic resonance imaging, ultrasonic imaging, photo-acoustic imaging and Raman spectroscopic imaging. Imaging equipment has its advantages and disadvantages respectively. Nuclear imaging systems have high sensitivity, but low resolution. In addition, using radioactivity has legal restrictions. In the case of magnetic resonance imaging systems, they have high resolution, but low sensitivity. Optical imaging systems have superior sensitivity and provide imaging faster than other imaging systems [3]. Because of its advantages, many researchers use optical imaging systems when undergoing pre-clinical tests. These imaging techniques are differently used to diagnosis a certain disease because imaging systems

\footnotetext{
${ }^{*}$ Corresponding author: Hee Joon Park, Department of Biomedical Engineering, Keimyung University, 1095 Dalgubeoldae-ro, Dalseo-Gu, Daegu, 702-701, Korea. Tel.: +82-53-580-3731. E-mail: hjpark@kmu.ac.kr.
} 
have different spectrums. Multi-imaging equipment is developed as combining different imaging systems nowadays [4].

NIR wavelength range is from $700 \mathrm{~nm}$ to $1000 \mathrm{~nm}$. The use of NIR light for biomedical imaging is grounded in first principles and is best understood in the context of photon propagation through living tissue and the signal to noise ratio. Generally, the photon absorbance of a particular tissue or organ is all-absorbing components. In living, non-pigmented tissue, the major NIR absorbers are water, lipids and hemoglobin. In a typical tissue, having $8 \%$ blood volume and $29 \%$ lipid content, the dominant absorber is hemoglobin, accounting for 39 63\% of the total absorbance at NIR wavelengths [5].

In order to develop imaging equipment, the researchers have to consider fluorescent agents properties, tissue targeting and selectivity of emitted fluorescent agents etc. In this study, new imaging equipment using visible ray and near-infrared ray for pre-clinical tests with small animals was developed. This equipment can provide a visible image, NIR image emitting fluorescent agents and a merged image simultaneously. It helps the researcher to understand visible messages. A communication system was specifically established to check obtained images through a smart pad in real time. Also, this equipment was made portable to move easily.

\section{Experimental}

\subsection{Design of the visible and NIR imaging equipment for small animals}

The schematic diagram of equipment is shown in Figure 1. In this equipment, a CCD camera was used to take visible and NIR images. A focusing lens and an objective lens are installed under a CCD camera. In order to pass only NIR light source, a NIR band pass filter was used, which is equipped with an automatic driving motor. There are NIR light sources for fluorescent agents around an object bed and visible light sources for illuminating an object under a ceiling. An object bed can move up and down with z-axis stepping motor (Figure 1).

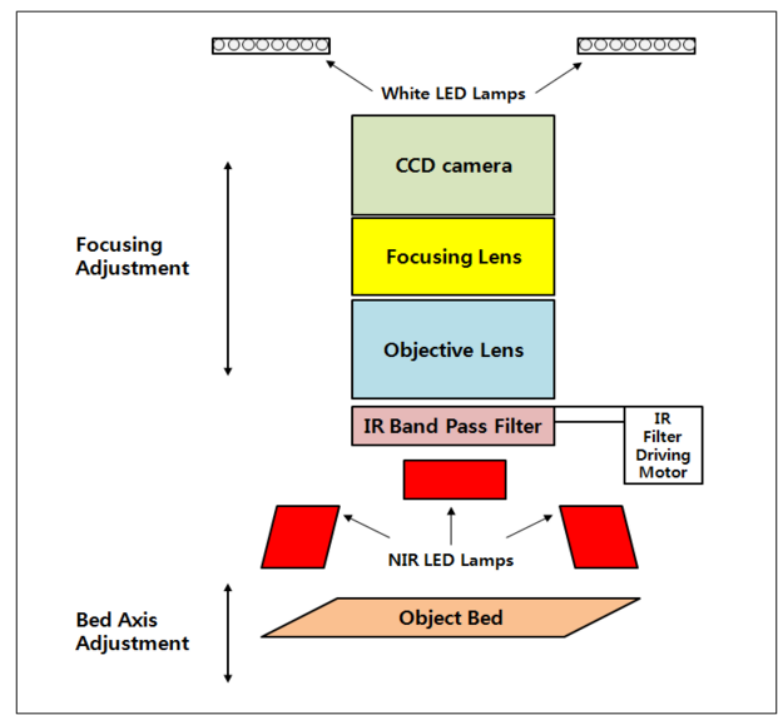

Fig. 1. Schematic diagram of portable visible and NIR imaging equipment. 
In the optical system, a CCD camera has a pixel size of $4.40 \times 4.40 \mu \mathrm{m}$ and the resolution is 1800 (H) $\times 1200$ (V) pixels (Figure 2(a)). The focal distance of the lens is from $8 \mathrm{~mm}$ to $80 \mathrm{~mm}$, and the zoom ratio is 10 (Figure 2(b)). IR band pass filter receives $850 \mathrm{~nm}$ wavelengths (Figure 2(c)). The white LEDs were used for a visible light source (Figure 2(d)), and an NIR light source emitting 750 $\mathrm{nm}$ wavelength for a NIR light source (Figure 2(e)). Each light source is fixed to a fabricated jig and light on the subject. Light sources are used to emit light of two wavelengths simultaneously or selectively.

To observe visible and NIR images, an imaging program was developed. Obtained images are shown on the computer screen through the imaging program. There are still cut images, visible images, NIR images and merged images on one screen. A merged image is an overlapped image of visible and NIR. It will be helpful for a researcher to understand visible messages.

\subsection{Preparation of the PLGA/HSA/ICG nanoparticles for NIR images}

In this study, nanoparticles were prepared by following the double emulsion method [6,7]. The nanoparticles are used by using the NIR image using the equipment. ICG as NIR fluorescent agents was encapsulated into the PLGA by using the double emulsion method. The PLGA is a biocompatible and biodegradable polymer that was approved by the Food Drug Administration (FDA). A preparation scheme of nanoparticles is shown in Figure 3.

In a double emulsion (W1/O/W2) method, PLGA $(100 \mathrm{mg})$ were dissolved in methylene chloride (2 $\mathrm{ml})$. ICG $(5 \mathrm{mg})$ and HSA $(15 \mathrm{mg})$ were dissolved in water $(250 \mathrm{ml})$. An aqueous solution containing ICG-HSA complex was emulsified in PLGA solution by the high-speed homogenizer for $5 \mathrm{~min}$. The resulting primary emulsion was added to PVA $(4 \%, \mathrm{w} / \mathrm{w} ; 30 \mathrm{ml})$ and emulsified using micro-tip probe sonicator (CV18, 700W, $20 \mathrm{kHz}$, SONICS\&MATERIALS, USA) set 90\% power for $5 \mathrm{~min}$. The resultant double emulsion was agitated with a magnetic stirrer overnight at room temperature until the evaporation of methylene chloride was complete. The PLGA/HSA/ICG nanoparticles were collected by ultracentrifugation $(17,000 \mathrm{rpm}, 10 \mathrm{~min})$, washed three times with distilled water, freeze-dried, and stored at $4^{\circ} \mathrm{C}$ before use.

\subsection{Characterization of the PLGA/HSA/ICG nanoparticles}

The morphology of the PLGA/HSA/ICG nanoparticles was obtained by using a field-emission scanning electron microscopy (SEM; S-4300, Hitachi, Japan). Dynamic light scattering particle size analyzer (N5, Beckman Coulter) was used to measure average diameter of the PLGA/HSA/ICG nanoparticles. The fluorescence property of the PLGA/HSA/ICG nanoparticles and ICG was measured by using a luminescence spectrometer (LS45, Perkin-Elmer, USA). The emission of PLGA/HSA/ICG nanoparticles and ICG were measured at $780 \mathrm{~nm}$.

\subsection{Observation of visible and NIR images}

In order to observe images, PLGA/HSA/ICG nanoparticles $(5 \mathrm{mg})$ in PBS buffer (pH 7.4, $1.5 \mathrm{ml}$ ) and distilled water were prepared in Eppendorf tubes respectively. For animal testing, PLGA/HSA/ICG nanoparticles $(0.5 \mathrm{mg})$ in PBS buffer $(\mathrm{pH} 7.4,1.5 \mathrm{ml})$ were prepared. Korea Research Institute of Bioscience and Biotechnology (KRIBB) were asked to conduct animal testing. An Eight-week-old male hairless mouse (Orient Bio, Seongnam, Korea, $31.8 \mathrm{~g}$ ) were anesthetized by Zoletil 50 (Virvbac, France, $0.08 \mathrm{ml} / \mathrm{kg}$ ) of $0.025 \mathrm{ml}$ through an intramuscular injection. In vivo behavior 
of the PLGA/HSA/ICG nanoparticles can be observed from real-time imaging after a subcutaneous injection to a tail of a mouse.

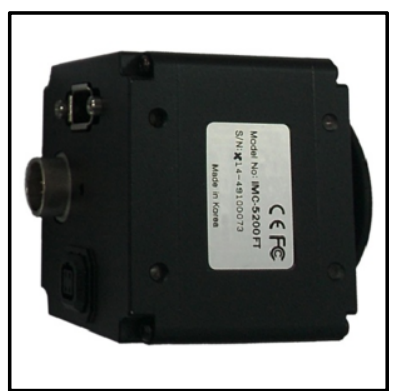

(a)

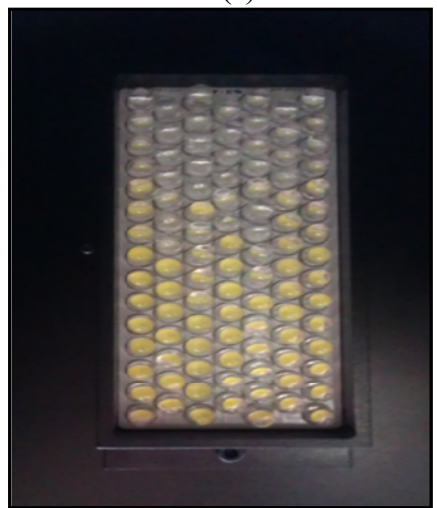

(d)

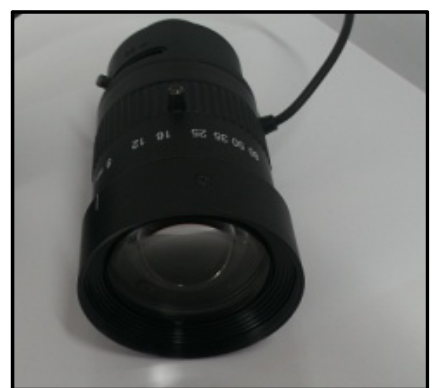

(b)

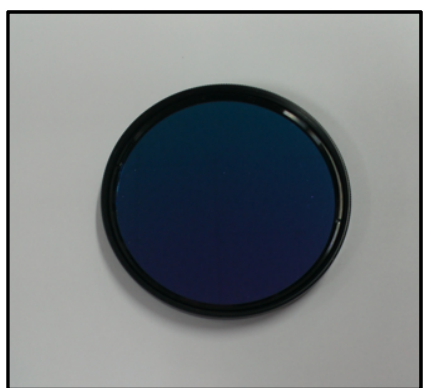

(c)

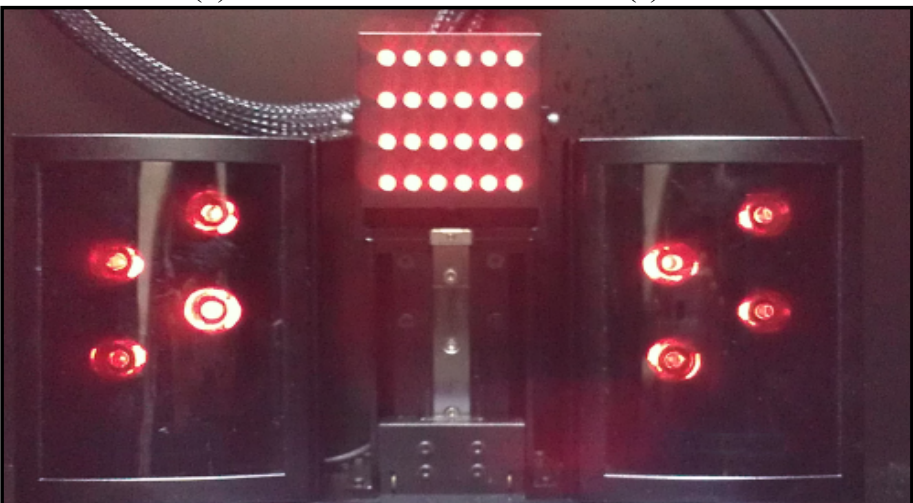

(e)

Fig. 2. Components of the optical system: (a) CCD camera (b) Lens (c) NIR band pass filter (d) Visible LED array light source (e) NIR LED array light source.
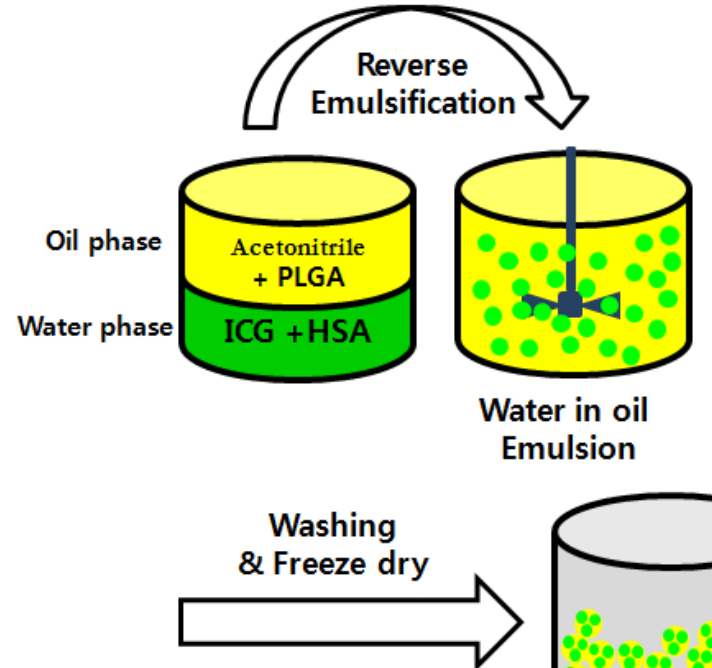

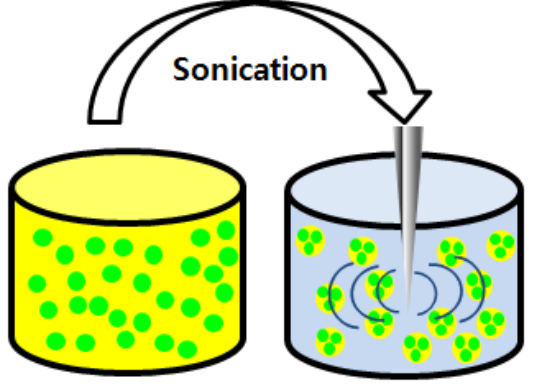

Emulsifier solution

Double (Water in oil in water) Emulsion

Fig. 3. Nanoparticle fabrication using double emulsion method. 
Animal care and all experiments were conducted in accordance with KRIBB Guidelines for the Care and Use of Laboratory Animals, and all experiments were approved by the institutional review board (KRIBB Institutional animal care and use committee/KRIBB-IACUC).

\section{Results}

\subsection{Visible and NIR imaging equipment for small animals}

The visible and NIR imaging equipment for small animals is shown in Figure 4(a). Outer size of the equipment is $\mathrm{W} 380 \times \mathrm{D} 720 \times \mathrm{H} 420$, and the inner size is $\mathrm{W} 300 \times \mathrm{D} 370 \times \mathrm{H} 330$. Also, the size of an object bed is W200 $\times \mathrm{H} 200 \times \mathrm{T} 2$. The object bed can move up and down with z-axis stepping motor. The weight of the equipment is $45 \mathrm{~kg}$. The equipment inside is shown in Figure 4(b). A CCD camera is fixed to observe the center of an object bed. A focusing lens and an objective lens are installed to adjust the image. There are NIR light sources around an object bed and visible light sources under the ceiling. In order to pass only NIR light sources, a NIR band pass filter was used, which is equipped with an automatic driving motor. It is convenient that an object bed can move up and down using a computer system.

A camera is possible to communicate with a laptop computer using 1394A port. A laptop computer is connected to 1394A 4pin ports. In that case, it can directly be connected to the camera. A laptop computer having no connection ports is used after installing IEEE 1394PCMCIA card.

A visible image, NIR image, merged image and a still cut image were obtained simultaneously. Especially, the NIR image was taken according to control the NIR band pass filter. Each image is displayed on the screen respectively, and a full screen is available for use. There are still cut images (Figure 5(a)), visible images (Figure 5(b)), NIR images (Figure 5(c)) and merged images (Figure 5(d)) on the screen. Also, the imaging program was developed to increase sensitivity. The imaging program can adjust the exposure time and the gain value of a CCD camera.

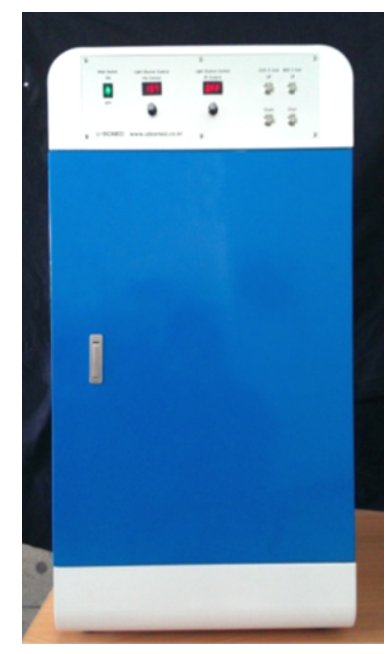

(a)

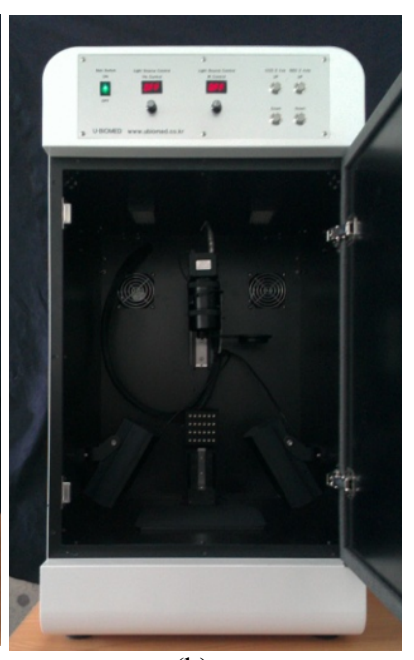

(b)

Fig. 4. Portable Visible and NIR imaging equipment (a) Outline of equipment (b) Configuration of system inside. 


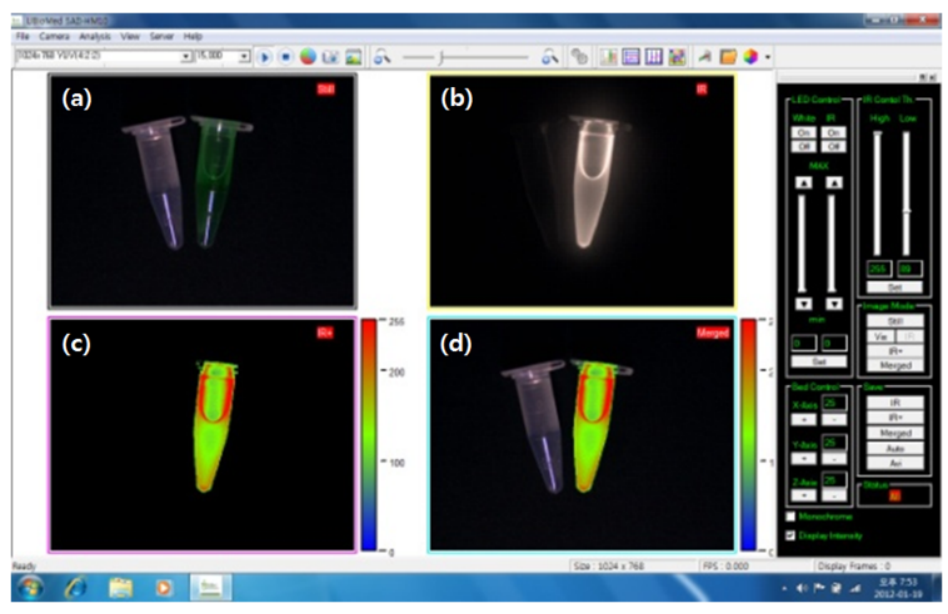

Fig. 5. Imaging viewer for Visible, NIR, and Merged image.

The resolution was set properly to a laptop computer on $1024 \times 768$ considering the laboratory environment and user convenience. Also, there are no restrictions to adjust the resolution on the imaging program. The imaging program will automatically save the data undergoing experiments to prevent data loss. It has an additional function that images for transmission can be automatically saved and deleted. Images can be sent to homepage servers to monitor the experiment remotely.

\subsection{Characterization of the PLGA/HSA/ICG nanoparticles}

The morphology of the PLGA/HSA/ICG nanoparticles is shown in Figure 6 using a field-emission scanning electron microscopy (SEM; S-4300, Hitachi, Japan). The PLGA/HSA/ICG nanoparticles have sphere morphology. The average diameter of the PLGA/HSA/ICG nanoparticles is approximately $184 \mathrm{~nm}$. ICG have emission peak at a wavelength of $820 \mathrm{~nm}$ and emission peak of the PLGA/HSA/ICG nanoparticles was observed at $810 \mathrm{~nm}$ (Figure 7).

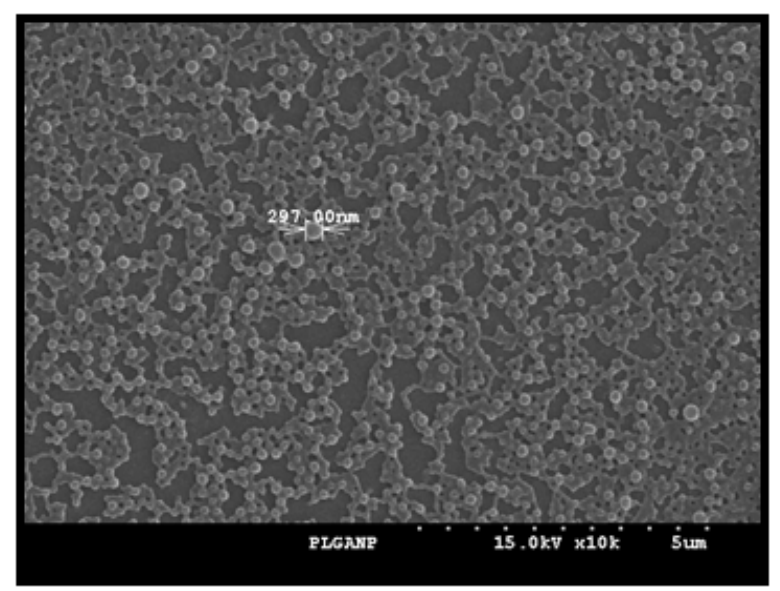

Fig. 6. SEM image of PLGA/HSA/ICG nanoparticles. 


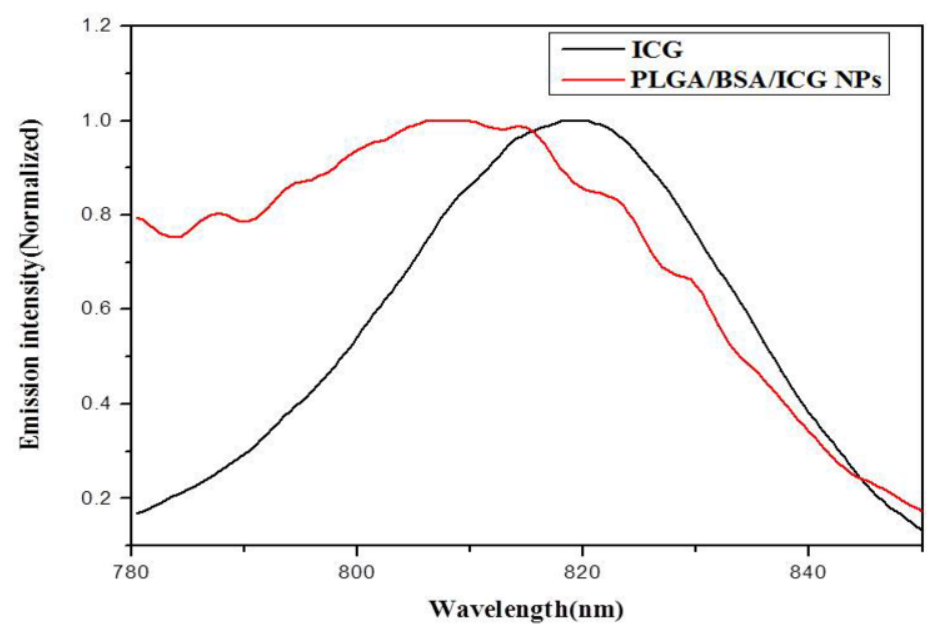

Fig. 7. Emission spectra of ICG and PLGA/HSA/ICG nanoparticles.

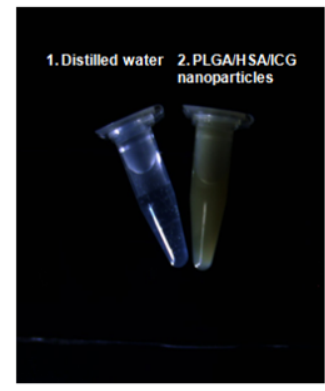

(a)

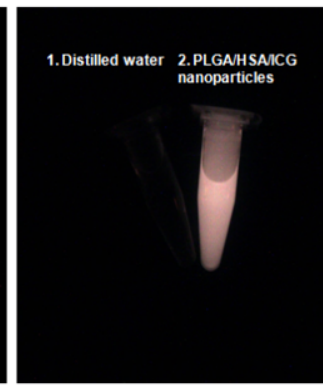

(b)

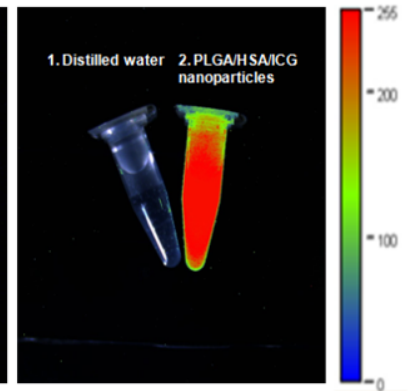

(c)

Fig. 8. Images of distilled water (left) and PLGA/HSA/ICG nanoparticles solution (right) (a) Color image (b) NIR image, and (c) Merged image.

\subsection{Observation of visible and NIR images}

The PLGA/HSA/ICG nanoparticles solution and distilled water in Eppendorf tubes respectively were prepared on an object bed and observed by using the visible and NIR imaging program as shown in Figure 8. We adjusted the value of the CCD camera through the developed imaging program. Exposure time was 1.7 seconds and gain value was 150 .

Figure 8(c) shows that the merged image shows intensity of the PLGA/HSA/ICG nanoparticles with color. The color change indicates emission change of the PLGA/HSA/ICG nanoparticles.

A mouse was fixed on an object bed and observed by using the visible and NIR imaging program as shown in Figure 9. The images can observe real-time distribution of the PLGA/HSA/ICG nanoparticles after a subcutaneous injection in the tail of a mouse. These images mean a dispersion of nanoparticles and release ICG from the PLGA/HSA/ICG nanoparticles (Vishal et al., 2009). Exposure time was 3.5 seconds and gain value was 150 . 


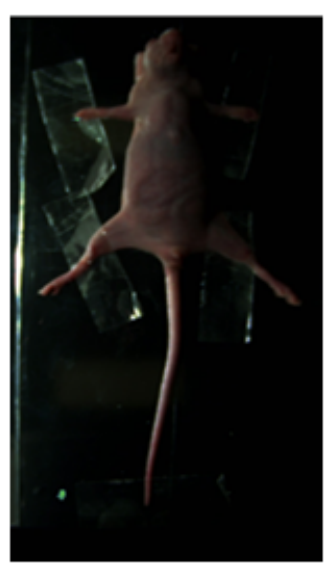

(a)

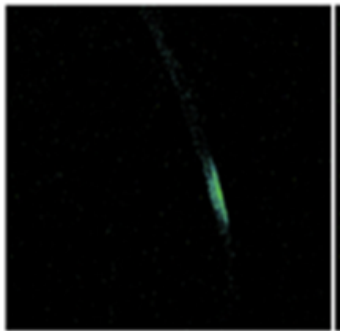

(d)

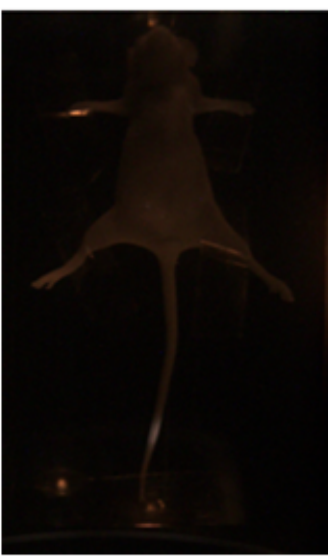

(b)

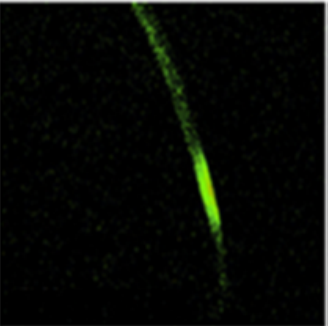

(e)

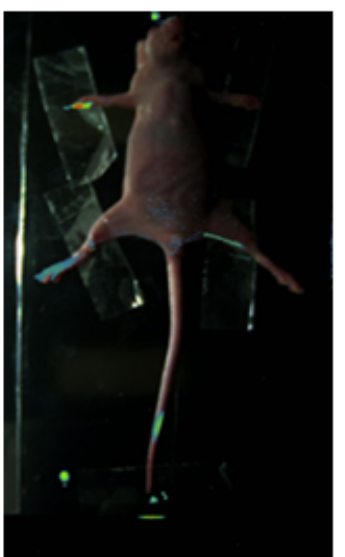

(c)

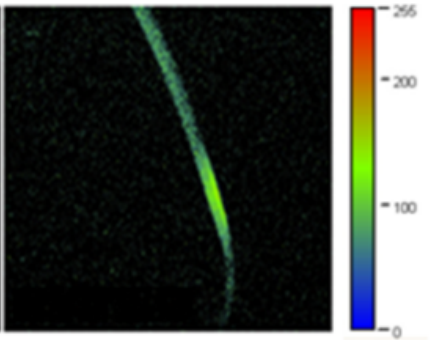

(f)

Fig. 9. Image of PLGA/HSA/ICG nanoparticles by tail subcutaneous injection of hairless mouse; (a) Color image (b) NIR image (c) Merged image (d) $5 \mathrm{~min}$ after injection (e) 30min after injection (f) $60 \mathrm{~min}$ after injection.

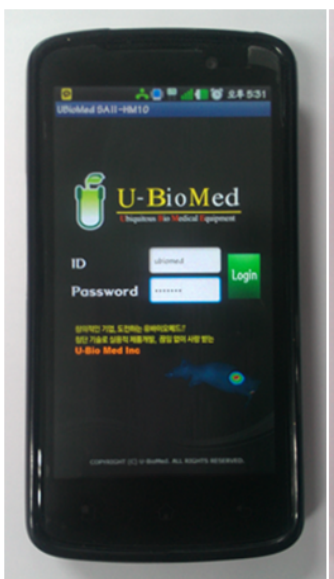

(a)

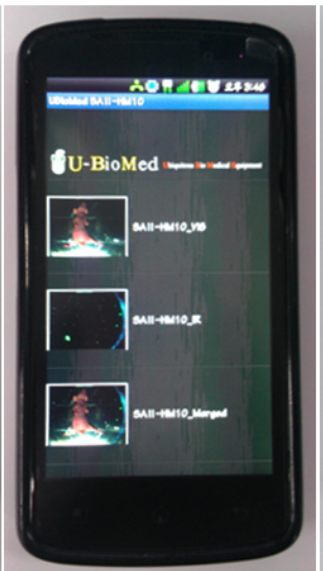

(b)

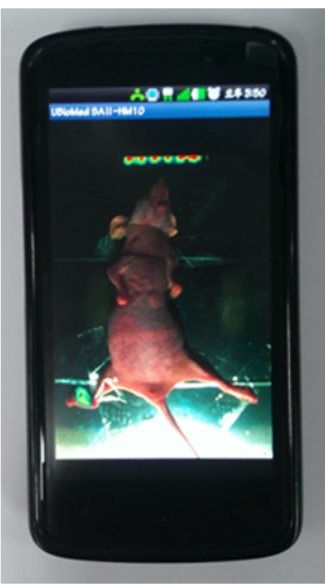

(c)

Fig. 10. Images through a smart phone application; (a) Login screen of the application (b) Screen of an imaging test using the application (c) Screen of the merged image.

Figure 9(a) is a color image, Figure 9(b) is a NIR image and Figure 9(c) is a merged image of the above two images. Figure $9(\mathrm{~d})$ is a merged image after injecting the PLGA/HSA/ICG nanoparticles. 
Figure 9(e) is 30 minutes after injection and Figure 9(f) is 60 minutes after injection. As a result, it is possible to track in vivo behavior of PLGA/HSA/ICG nanoparticles using this equipment.

Finally, obtained visible and NIR images were observed by using a smartphone (LG-SU640, LG, Korea) through the application program. The figure running the application program is shown in Figure 10(a). In the Figure 10(b), visible, NIR and merged image can be observed on one screen. Figure $10(\mathrm{c})$ is a zoom in the image after selecting the merged image.

\section{Conclusion}

In this study, a portable visible and NIR imaging equipment was developed for pre-clinical tests with small animals. The advantage of the equipment is its ability to communicate with researchers who are a long distance away. To be specific, the experimental information can be shared by smart phones in real time. Therefore, researchers can save time and money when conducting a pre-clinical test. As visible light sources and NIR light sources are harmless to humans, the equipment has great potential and application ranges which can be gradually expanded.

\section{Acknowledgement}

This research was supported by the MOTIE (Ministry of Trade, Industry and Energy), Korea, under the Inter-Economic Regional Cooperation program (R0002625) supervised by the KIAT (Korea Institute for Advancement of Technology).

\section{References}

[1] Colburn, W. A., Biomarkers in drug discovery and development: From target identification through drug marketing. J Clin Pharmacol 43(4), 329-41, 2003.

[2] Homer H Pien, Alan J Fischman, James H Thrall, A Gregory Sorensen, Using imaging biomarkers to accelerate drug development and clinical trials. Drug Discov Today 2005; 10(4):259-66, 2005.

[3] Lim YT, Cho MY, Noh YW, Chung JW, Chung BH, Near-infrared emitting fluorescent nanocrystals-labeled natural killer cells as a platform technology for the optical imaging of immunotherapeutic cells-based cancer therapy, Nanotechnology, 20(47):475102, 2009.

[4] Herbert Kaplan, Practical Applications of Infrared Thermal Sensing and Imaging Equipment, $3^{\text {rd }}$ Ed, SPIE, 2007.

[5] J.V Frangioni, In vivo near-infrared fluorescence imaging, Current Opinion in Chemical Biology 7 (2005), 626-634

[6] M.F Zambauxa, et al., Influence of experimental parameters on the characteristics of poly(lactic acid) nanoparticles prepared by a double emulsion method, Journal of Controlled Release, 50(1-3), 31-40, 1998. ..

[7] Y.-T. Lim, Y.-W. Noh, J.-H. Han, Q.-Y. Cai, K.-H. Yoon and B.-H. Chung, Biocompatible polymer-nanoparticle-based bimodal imaging contrast agents for the labeling and tracking of dendritic cells, Small 4 (2008), 1640-1645. 